\title{
An Effective Method to Prepare Polymer/Nanocrystal Composites with Tunable Emission over the Whole Visible Light Range
}

\author{
Haotong Wei ${ }^{1}$, Haizhu Sun ${ }^{1,2}$, Hao Zhang ${ }^{1}$, Cong Gao ${ }^{1}$, and Bai Yang ${ }^{1}(\bowtie)$ \\ ${ }^{1}$ State Key Laboratory of Supramolecular Structure and Materials, College of Chemistry, Jilin University, Changchun 130012, China \\ ${ }^{2}$ College of Chemistry, Northeast Normal University, Changchun 130024, China \\ Received: 27 April 2010 / Revised: 18 May 2010 / Accepted: 19 May 2010 \\ C The Author(s) 2010. This article is published with open access at Springerlink.com
}

\begin{abstract}
Materials with emission over the whole visible range $(400-800 \mathrm{~nm})$ have been obtained through incorporating single-colored CdTe nanocrystals (NCs) into a poly( $p$-phenylene vinylene) (PPV) precursor [the sulfonium polyelectrolyte precursor of PPV]. Firstly, the quantum yield (QY) of the PPV precursor was improved to $~ 50 \%$ via heat treatment of a mixed solution of the PPV precursor and poly(vinyl alcohol) (PVA) at $100{ }^{\circ} \mathrm{C}$ for $3 \mathrm{~min}$. Then, single-colored CdTe NCs were incorporated into the mixed solution. The introduction of the PVA was necessary to reduce the electrostatic interaction between the PPV precursor and CdTe NCs, which improved their compatibility. The reduced electrostatic interaction eliminated Förster resonance energy transfer (FRET) processes between NCs, as well as between NCs and the PPV precursor, which allowed the functional integration of the polymer and NCs. Consequently, polymer/NC composites with almost any Commission Internationale de L'Eclairage (CIE) coordinates can be achieved by simply changing the size and amount of the NCs. In particular, when the emission wavelength of the CdTe NCs was $559 \mathrm{~nm}$, a pure white-light emitting material with CIE coordinates $(0.337,0.332)$ was obtained.
\end{abstract}

\section{KEYWORDS}

Nanocrystal, poly(p-phenylene vinylene), CdTe, poly(vinyl alcohol)

\section{Introduction}

Aqueous semiconductor nanocrystals (NCs) are known for their tunable emission spectra spanning the UV to near-infrared regions, high photoluminescence quantum yields (PLQYs), and biocompatibility. Therefore, they are currently under intensive investigation for their potential applications in quantum-dot lasers [1-3], light emitting diodes [4-6], biological labeling [7-13], and other areas. For example, large optical gain has been obtained by Klimov and coworkers using chemically synthesized NCs, which allows the application of these NCs in the field of quantum-dot lasers [3]. Qian et al. synthesized water-soluble $\mathrm{CdHgTe} / \mathrm{CdS} \mathrm{NCs}$ with photoluminescence (PL) emission at 600-830 nm for imaging in living animals [10]. In spite of the achievements obtained with NCs, there are still some disadvantages, such as poor processability and instability, which limit their further development and applications.

Many groups have explored the use of polymer materials as a way of improving the properties of

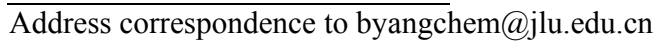


NCs, but two problems remain. One is how to ensure the compatibility between NCs and polymers, because NCs easily become aggregated due to their large specific surface area. In our previous research [14], amphiphilic surfactants with double bonds were used to transfer NCs from an aqueous phase into an oil phase by means of electrostatic forces, and then styrene copolymerized with the surfactant on the surface of the NCs. The NCs in this system acted as cross-linking points and consequently, their stability and the compatibility between the NCs and the polymer were both enhanced. The other is how to tune and integrate the properties of the NCs and the polymer, including the mechanical properties, optical properties, and smart response properties. For example, thermosensitive poly( $\mathrm{N}$-isopropyl acrylamide) microgels covered with CdTe NCs have been prepared by Rubio-Retama et al. [15], in which the stability of the NCs was enhanced and simultaneously the thermal response function was realized.

The preparation of white-light emitting materials has developed rapidly in recent years due to their applications in full color displays and illumination sources. Various kinds of polymer have been synthesized in order to prepare white-light emitting materials [16-19]. Normally, blue, green, and red are the basic colors required to obtain white-light emission $[20,21]$. Two complementary color molecules are also good choices [22-24]. For example, Wang et al. prepared a single-polymer white-light system involving two-colored chromophore emission [24]. Many groups have developed other methods to prepare NCs with white-light emission without using polymers, because the process of preparation is generally easier [25]. However, the problem is that traditional whitelight emission needs the usage of multi-colored NCs, which results in the Förster resonance energy transfer (FRET) between NCs, and consequently the efficiency of the materials remains relatively low. Recently, CdSe NCs and Mn-doped ZnS NCs have been synthesized and gave high quality white-light emission [26, 27]; although the FRET process was eliminated, the stability remains a problem to be solved. Therefore, the preparation of white-light emitting materials using NCs remains a challenge.
Poly(p-phenylene vinylene) (PPV) and its derivatives are important functional polymers which have found applications in transistors, photovoltaic devices, and light-emitting diodes [28-33]. However, little attention has been paid to the PPV precursor [the sulfonium polyelectrolyte precursor of PPV], which is water soluble, has a high quantum yield (QY) and is easy to process. Above all, the PPV precursor is one kind of polyelectrolyte which can be used as a building block during the electrostatic assembly process. In this work, we investigated the PL properties of the PPV precursor, concentrated on how to integrate the PL properties of the PPV precursor and CdTe NCs in order to obtain emission over the whole visible range, and how to realize the controllable regulation of the emitted color. We demonstrate that emission over the whole visible range can be easily obtained by tuning the size and amount of the NCs. A PPV precursor/CdTe NCs/poly (vinyl alcohol) (PVA) composite solution giving pure white-light emission with the Commission Internationale de l'Eclairage (CIE) chromaticity coordinates $(0.337,0.332)$ was prepared, and the mechanism of the white-light emission process is discussed in detail.

\section{Experimental section}

\subsection{Preparation of the PPV precursor}

The preparation of the PPV precursor can be found elsewhere [28-33]. Briefly, $10 \mathrm{~mL}$ of $0.4 \mathrm{~mol} / \mathrm{L} \mathrm{NaOH}$ was added to $10 \mathrm{~mL}$ of $0.4 \mathrm{~mol} / \mathrm{L} p$-xylylenebis (tetrahydrothiophenium chloride) solution in methanol. This solution was cooled to $0-5{ }^{\circ} \mathrm{C}$ in an ice bath. The reaction was allowed to proceed for $1 \mathrm{~h}$ and was then terminated by the addition of $0.1 \mathrm{~mol} / \mathrm{L} \mathrm{HCl}$ aqueous solution to neutralize the reaction solution. The PPV precursor aqueous solution was dialyzed against deionized water for one week. The structure of the PPV precursor is given in Scheme S-1 in the Electronic Supporting Material (ESM).

\subsection{Preparation of CdTe NCs}

The precursor solution of CdTe NCs was prepared by adding freshly prepared NaHTe solution into a

\section{型 Springer}


$1.25 \times 10^{-3} \mathrm{~mol} / \mathrm{L} \mathrm{N}_{2}$-saturated $\mathrm{CdCl}_{2}$ solution at $\mathrm{pH} 9.0$ in the presence of 3-mercaptopropionic acid (MPA) acting as a stabilizing agent. The precursor solution was then subjected to a reflux in order to control the growth of CdTe NCs. The molar ratio of $\mathrm{Cd}^{2+} / \mathrm{MPA} /$ $\mathrm{HTe}^{-}$was 1:2.4:0.2.

2.3 Manipulation of the color emitted by the composite solution

The mass fraction of the PVA-2099 $\left(M_{n}=88000,99 \%\right.$ hydrolyzed) solution was $10 \%$ and the concentration of the PPV precursor solution was about $0.1 \mathrm{wt} . \%$. The PVA solution was mixed with the PPV precursor solution with a mass ratio of $7: 3$. Then the hybrid was heated at $100{ }^{\circ} \mathrm{C}$ for a few minutes in order to increase the QY of the solution followed by addition of the above CdTe NCs solution. By changing the size of CdTe NCs and amount of the CdTe NCs solution, we could control the color emitted as well as the chromaticity coordinates.

\subsection{Characterization}

UV-vis absorption spectra were recorded using a Shimadzu 3100 UV-vis-near-IR spectrophotometer. Fluorescence spectra were acquired on a Shimadzu RF-5301 PC spectrofluorometer. The excitation wavelength was $365 \mathrm{~nm}$. The PLQY of NCs and composite solution were estimated at room temperature using quinine in aqueous $0.5 \mathrm{~mol} / \mathrm{L} \mathrm{H}_{2} \mathrm{SO}_{4}$ as $\mathrm{PL}$ reference. The luminescence lifetime were recorded on a HORIBA Jobin Yvon FL3-TCSPC spectrophotometer with a time resolution of about 100 ps. All optical measurements were performed at room temperature under ambient conditions. Zeta potential measurements were performed using a Zetasizer Nano-ZS (Malvern Instruments). Transmission electron microscopy (TEM) images were recorded on a JEOL-2010 electron microscope operating at $200 \mathrm{kV}$. Fourier transform infrared (FT-IR) spectra were recorded with a $\mathrm{KBr}$ window on a Nicolet Avatar 360 FT-IR spectrophotometer. The color of light was identified by the CIE (Commission Internationale de l'Eclairage 1931) colorimetry system. Any color can be described by the chromaticity $(x, y)$ coordinates on the CIE diagram (an example is shown in Fig. 6).

\section{Results and discussion}

\subsection{PL Properties of the PPV precursor}

The structure of the PPV precursor is described in the ESM. Normally, the elimination reaction takes place in the side group of the PPV precursor which often results in a conjugated scaffold under ambient conditions, leading to blue-light emission [28]. We monitored the change in the PL properties of the PPV precursor with storage time. It can be seen from Fig. 1 that the shapes of both the UV absorption and PL emission spectra show little change during the five weeks after preparation. The maxima are always located at $315 \mathrm{~nm}$ for the UV absorption and $430 \mathrm{~nm}$ for the PL emission spectra. This indicates that the electronic structure of the PPV precursor remains unchanged over time. The QYs of the PPV precursor after different storage times are shown in Table 1. The QY is $31.2 \%$ for the freshly prepared material, increases to $40.4 \%$ after one week, then decreases to $38.2 \%$ after two weeks, and reaches a plateau during the following weeks. We think the elimination reaction has two effects on the PL properties of the PPV precursor. One is that the increased content of partly conjugated chains results in the enhancement of the QY. The other is when a large number of conjugated chains are generated, the $\pi-\pi$ conjugated building blocks approach each other, and lead to the decrease of the QY. These two effects counteract each other and result in the plateau for the change of QY. The presence of the interchain interactions in the PPV precursor is confirmed by the PL spectrum in Fig. 1(b). A shoulder peak located at $\sim 460 \mathrm{~nm}$, although it is not particularly marked, can be assigned to the interchain interaction [34-37] while the main peak is assigned to the single-chain exciton recombination. As a result, the emission may involve single or multiple conjugated chains which are not spectrally distinguishable. The same is true for the absorption peak located at $370 \mathrm{~nm}$. Therefore on storing, the QY of the PPV precursor initially increases, decreases after two weeks and remains almost unchanged during the following weeks $[38,39]$. 
Table 1 The PLQY of the PPV precursor and the PPV precursor/ PVA blends before and after being heated. The PPV precursor was stored below $4{ }^{\circ} \mathrm{C}$ and measurements were made under ambient conditions

\begin{tabular}{cccc}
\hline \multirow{2}{*}{$\begin{array}{c}\text { Time } \\
\text { (weeks) }\end{array}$} & $\begin{array}{c}\text { QY of the PPV } \\
\text { precursor (\%) }\end{array}$ & \multicolumn{2}{c}{ QY of the PPV precursor/PVA (\%) } \\
\cline { 3 - 4 } & 31.2 & 23.9 & 54.5 \\
0 & 40.4 & 18.5 & 50.1 \\
1 & 38.2 & 32.2 & 47.1 \\
2 & 33.0 & 24.6 & 43.4 \\
3 & 32.6 & 22.4 & 44.6 \\
4 & 36.0 & 25.2 & 53.2 \\
5 & 37.0 & 27.4 & 48.8 \\
11 & & & \\
\hline
\end{tabular}

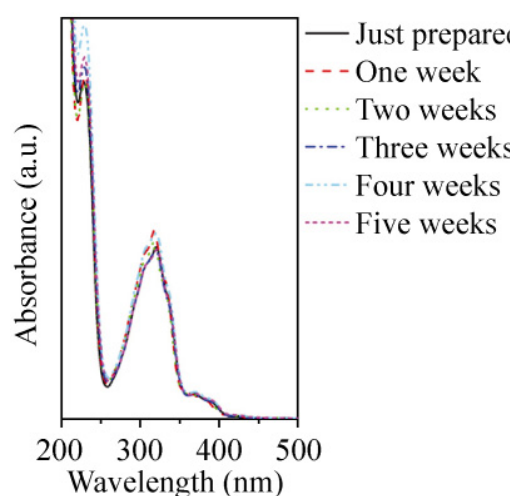

(a)

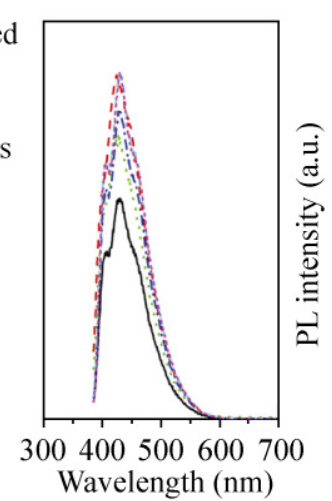

(b)
Figure 1 (a) UV-vis absorption and (b) PL spectra (excited at $365 \mathrm{~nm}$ ) of the PPV precursor after different storage times

\subsection{PL properties of the PPV precursor/PVA mixture}

In order to improve the compatibility between CdTe NCs and the PPV precursor, it is necessary to introduce PVA into the system. It can be seen from Fig. S-1 that the introduction of the PVA does not influence the peak shape and position of the PPV precursor, which shows that the electronic structure of the PPV precursor is not affected. However, it has an important influence on the QY of the PPV precursor. From Table 1, it can be observed that the QYs of the PPV precursor/PVA mixed solution at different storage times are all much lower than those of the PPV precursor itself after the same storage time. We heated the solution of the PPV precursor/PVA at $100{ }^{\circ} \mathrm{C}$ for $3 \mathrm{~min}$ and Table 1 clearly shows that the QYs are significantly improved after heating. As mentioned elsewhere [38, 39], this heating process on one hand results in the formation of the partly conjugated chains which leads to an improvement of QY. Furthermore, the $\pi-\pi$ stacking quenching effect is alleviated by the isolation of the PVA polymer chains. Therefore, we obtained higher QY for the PPV precursor/PVA mixed solution. This is confirmed by Fig. 2, which shows that the PL lifetime of the PPV precursor in the polymer blends are $\sim 800$ ps for $\tau_{1}$ and $\sim 2480$ ps for $\tau_{2}$ with $86.74 \%$ and $13.27 \%$ contribution to the PL, respectively. Here, $\tau_{1}$ is related to the single-chain radiative decay, while $\tau_{2}$ is related to the congregated structure as a result of the interchain interaction or simply a larger size of polymer domains $[40,41]$. The PL lifetime of single-chain radiative decay for the polymer blend extends to $\sim 1100$ ps $(84.97 \%)$ and that of the congregated structure extends to $\sim 3300 \mathrm{ps}$ $(15.03 \%)$ after heating. The contributions to the PL coming from single-chain radiative and congregated structure change only very slightly on heating, which shows that the form of the PPV precursor varies little on heating. The increase of the PL lifetime of singlechain radiative decay results in the improvement of the QY, while the increase of the PL lifetime of the congregated structure indicates the alleviation of the $\pi-\pi$ stacking quenching effect and also results in the enhancement of QY. Consequently, the QY improves by about $100 \%$ after the heat treatment, which is important in terms of obtaining a white-light emitting material.

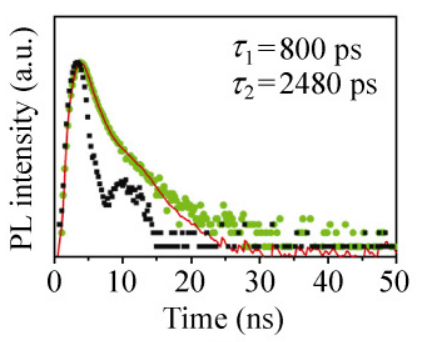

(a)

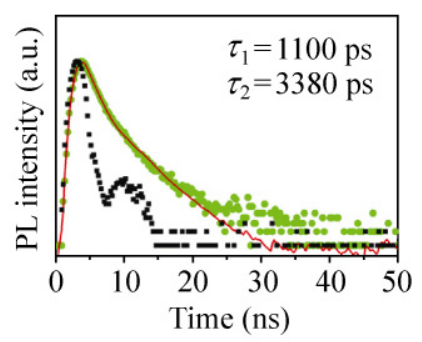

(b)
Figure 2 PL lifetime of solutions of (a) the PPV precursor in the presence of the PVA matrix before heating and (b) the PPV precursor in the presence of the PVA matrix after being heated, excited at $370 \mathrm{~nm}$, calculated by fitting the experimental data using the biexponential function $A_{1} \exp \left(\frac{t}{\tau_{1}}\right)+A_{2} \exp \left(\frac{t}{\tau_{2}}\right)$. Black squares: water as reference. Green circles: experimental data for the sample. Red line: fitted by the biexponential function 


\subsection{Introduction of CdTe NCs}

The combination of CdTe NCs and the PPV precursor is realized via electrostatic forces, as is confirmed by the change of zeta potential. The zeta potential of CdTe NCs is $-32.5 \mathrm{mV}$, and becomes $38 \mathrm{mV}$ after assembly with the PPV precursor. This process involves the decrease of the charge density on the surface of CdTe $\mathrm{NCs}$, causing the aggregation of CdTe NCs, and consequently resulting in the poor stability of the solution. An effective method to solve this problem is to introduce PVA into the system. In the FT-IR spectrum of the PPV precursor/CdTe NCs/PVA composite shown in Fig. 3(a), the peak located at $3300 \mathrm{~cm}^{-1}$ is assigned to the $-\mathrm{OH}$ of the PVA, and the peaks at $2940 \mathrm{~cm}^{-1}$ and $1097 \mathrm{~cm}^{-1}$ are assigned, respectively, to the $\mathrm{C}-\mathrm{H}$ stretching vibration and $\mathrm{C}-\mathrm{O}-\mathrm{C}$ stretching vibration resulting from the reaction of the PPV precursor and the PVA. The peak at $1655 \mathrm{~cm}^{-1}$ is assigned to the $\mathrm{C}=\mathrm{C}$ stretching mode and the peaks appearing between 600 and $900 \mathrm{~cm}^{-1}$ are caused by the Ar-C bond vibrations. This FT-IR spectrum confirms the presence of the PVA and that part of the PVA reacts with the PPV precursor to form $\mathrm{C}-\mathrm{O}-\mathrm{C}$ bonds. TEM image of the PPV precursor/CdTe NCs/PVA composite is shown in Fig. 3(b). The average size of the composite microspheres is about $40 \mathrm{~nm}$ as a result of assembly, which is larger than that of CdTe NCs. Figure 4 gives the PL lifetime of a pure solution of CdTe NCs, CdTe NCs in the PPV precursor/CdTe NCs/PVA composite and the PPV precursor in the PPV precursor/CdTe NCs/PVA composite. The PL lifetime associated with intrinsic combination of core states of CdTe NCs $\left(\tau_{1}\right)$ decreased from $10 \mathrm{~ns}$ to $3 \mathrm{~ns}$ while that $\left(\tau_{2}\right)$ associated with involvement of surface state changed from $38 \mathrm{~ns}$ to $28 \mathrm{~ns}$ after assembly in the composite solution. This

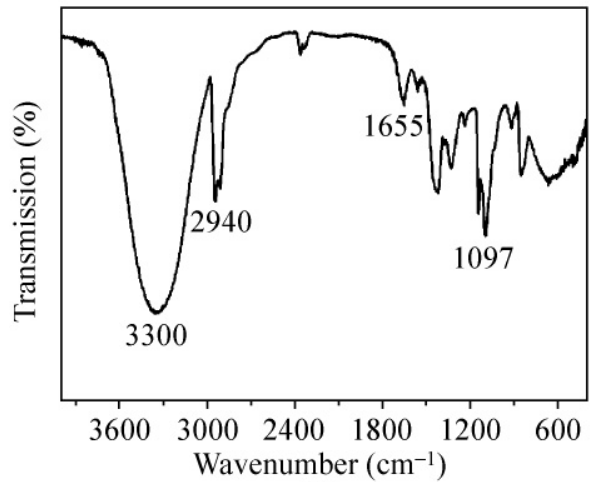

(a)

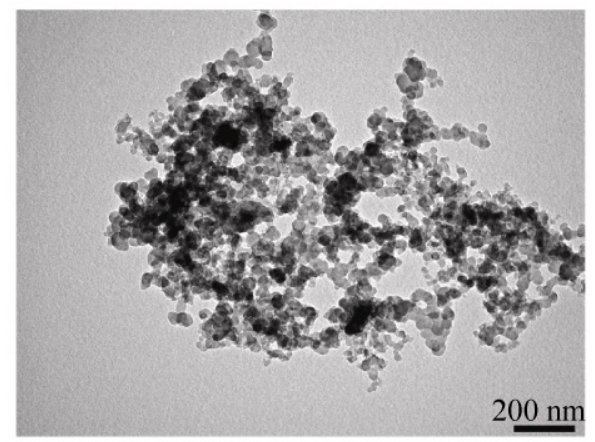

(b)

Figure 3 (a) IR spectrum and (b) TEM image of the PPV precursor/CdTe NCs/PVA composite

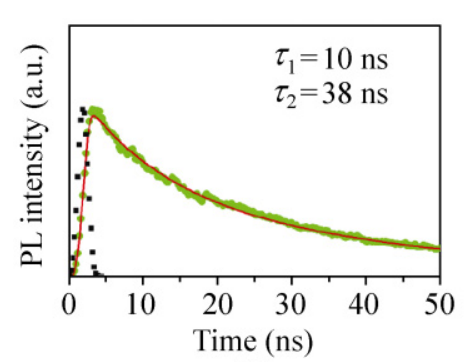

(a)

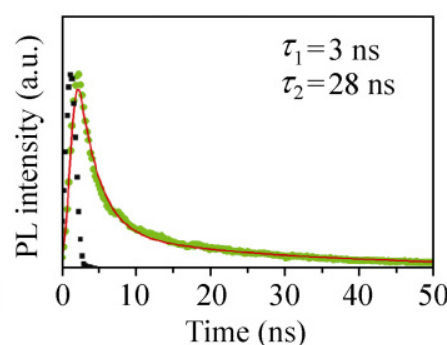

(b)

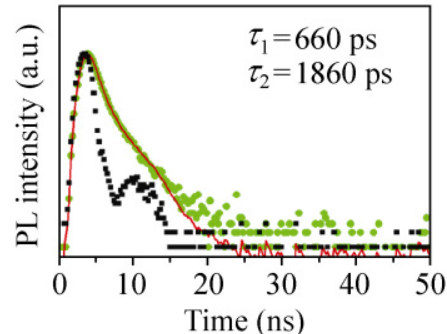

(c)

Figure 4 PL lifetime of (a) CdTe NCs solution, (b) CdTe NCs in the PPV precursor/CdTe NCs/PVA composite solution, (c) the PPV precursor in the PPV precursor/CdTe NCs/PVA composite solution, excited at $370 \mathrm{~nm}$, calculated by fitting the experimental data using the biexponential function $A_{1} \exp \left(\frac{t}{\tau_{1}}\right)+A_{2} \exp \left(\frac{t}{\tau_{2}}\right)$. Black squares: water as reference. Green circles: experimental data for the sample. Red line: fitted by the biexponential function 
indicates that the intrinsic interaction between $\mathrm{CdTe}$ NCs and polymer is still electrostatic, which often results in a decrease of PL lifetime $[42,43]$. The electrostatic interaction between NCs and the polymer can effectively stabilize the NCs in the polymer matrix when its strength is appropriate (as mentioned above, strong electrostatic interactions result in the aggregation of NCs). Through introducing PVA into the system, the appropriate strength of interactions is realized, which significantly improves the stability of the CdTe NCs/polymer system. From Fig. 4(c), it is observed that the PL lifetime $\left(\tau_{1}\right)$ of single-chain radiative decay for the polymer blend decreases to $\sim 660 \mathrm{ps}$ and that $\left(\tau_{2}\right)$ of the congregated structure decreases to $\sim 1860 \mathrm{ps}$ (26.56\%) which further confirms that there are electrostatic interactions between the NCs and the polymer. What is more, the contribution to the PL from the congregated structure of the PPV precursor changed from $15.03 \%$ to $26.56 \%$, confirming that its content increased in the composite solution. The decrease in PL lifetime of CdTe NCs, confirms there is no FRET occurring between polymer and NCs, which is a necessary condition for multi-color emission.

\subsection{Controllable regulation of the emitted color}

According to this simple method, we can prepare composite solutions with emission covering the whole visible light range including blue, cyan, green, yellow, orange, and red; photos are shown in Fig. 5(a). The UV spectra and PL spectra are presented in Figs. 5(b) and 5(c), the compositions of the solutions are summarized in Table 2, and the corresponding chromaticity coordinates are also listed in Table 2 . The blue light emission given by the PPV precursor/PVA mixture is a complement of the CdTe NCs. The other color-emitting solutions are composed of mixtures of the polymer and NCs. Although we obtained white-light emission

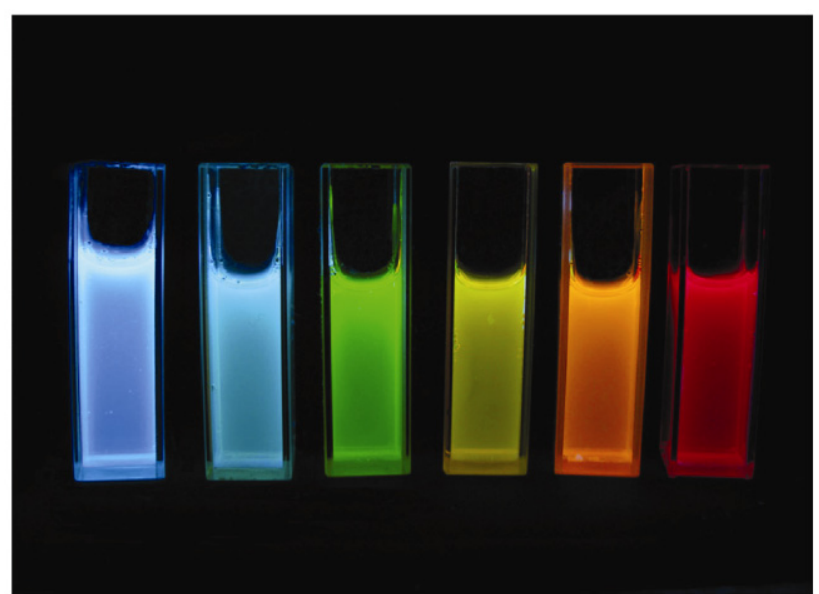

(a)

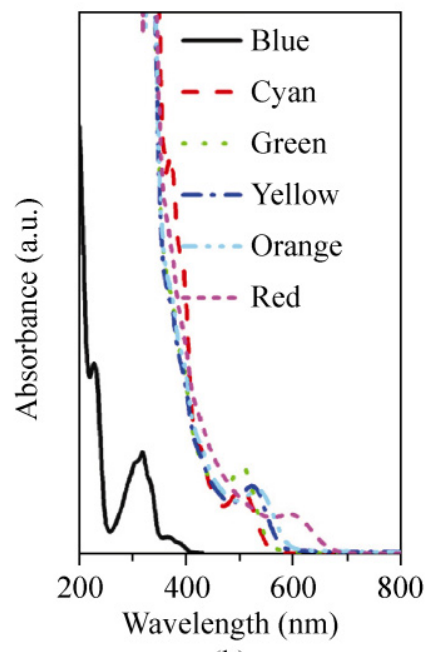

(b)

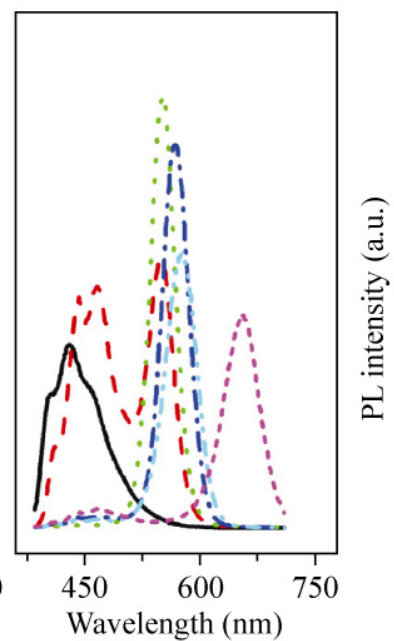

(c)
Figure 5 (a) Photos of composite solutions with different colors prepared with different sizes and amounts of CdTe NCs. (b) UV spectra and (c) PL spectra of the composite solutions

in our previous study [44], the observed CIE was $(0.27,0.37)$ which does not correspond to pure whitelight emission. Here, we systematically studied the emitted color by changing the emission wavelength and the amount of the CdTe NCs. We prepared a series of PPV precursor/CdTe NCs/PVA composite solutions

Table 2 The amounts of the PPV precursor/PVA mixture and NCs in the composite solutions with NCs having different emission wavelengths and the corresponding chromaticity coordinates

\begin{tabular}{lcccccc}
\hline \multicolumn{1}{c}{ Color } & Blue & Cyan & Green & Yellow & Orange & Red \\
\hline PPV precursor/PVA $(\mathrm{g})$ & 1 & 1 & 1 & 1 & 1 & 1 \\
Wavelength of CdTe $(\mathrm{nm})$ & $/$ & 534 & 534 & 550 & 559 & 620 \\
Amount of CdTe $(\mathrm{mL})$ & $/$ & 1.5 & 10 & 10 & 10 & 10 \\
Chromaticity coordinate & $(0.152,0.085)$ & $(0.209,0.311)$ & $(0.333,0.644)$ & $(0.423,0.540)$ & $(0.459,0.507)$ & $(0.545,0.289)$ \\
\hline
\end{tabular}


emitting different colors using NCs with emission wavelengths at 544,559, and $573 \mathrm{~nm}$. Figure 6 shows the CIE coordinates of the resulting composite solutions. As CdTe NCs are added to the mixed solution, the chromaticity coordinates gradually become further away from the blue region. What is most important is that there is a good linear relationship. The more CdTe NCs are added to the mixed system the further the shift in coordinates. When $544 \mathrm{~nm}$ CdTe NCs are added to the mixed solution, the chromaticity coordinate line appears in the green region, while for the $573 \mathrm{~nm} \mathrm{CdTe}$ $\mathrm{NCs}$, the chromaticity coordinate line appears in the red region. For CdTe NCs with emission wavelengths at $544 \mathrm{~nm}$ and $573 \mathrm{~nm}$, from Fig. 6, we can observe that it is impossible to obtain pure white-light emission whatever the amount of the CdTe NCs added. It can be concluded that only one kind of CdTe NCs with a specific emission wavelength can lead to pure whitelight emission. We selected CdTe NCs with emission wavelength at $559 \mathrm{~nm}$ and obtained pure white-light emission when the mass of NCs was about 8 times that of the PPV precursor. Figure 7 presents the UV-vis absorption and PL emission spectra of the white-light emitting solution. In the UV-vis spectrum, the absorption maxima located at $530 \mathrm{~nm}$ and $312 \mathrm{~nm}$ are assigned to CdTe NCs and the PPV precursor respectively, which shows that they coexist well in

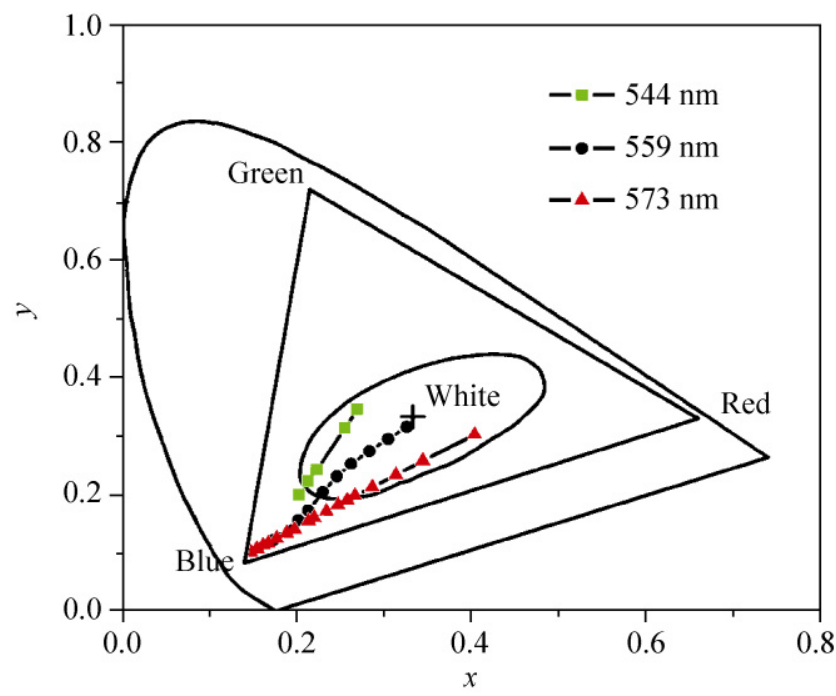

Figure 6 The chromaticity coordinate graph of the composite solutions prepared with CdTe NCs having different emission wavelengths. The chromaticity coordinate changes approximately linearly with increasing amount of the NCs

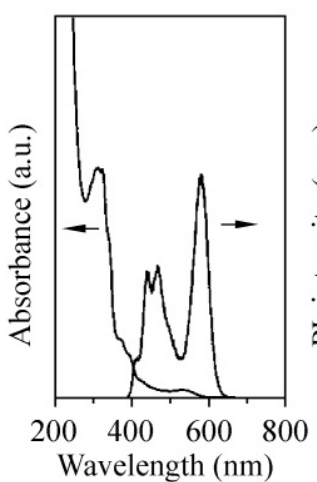

(a)

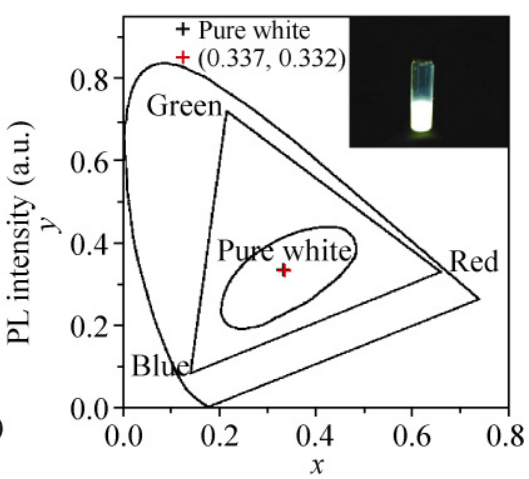

(b)
Figure 7 (a) UV-vis absorption and PL spectra (excited at $365 \mathrm{~nm}$ ) of the pure white-light emitting solution. (b) The chromaticity coordinates graph of the pure white-light emitting solution. The insert is a photo of the white-light emitting solution under ultraviolet radiation

the solution. In the PL spectrum the emission maxima located at $580 \mathrm{~nm}$ and $468 \mathrm{~nm}$ are attributed to the CdTe NCs and the PPV precursor, respectively, and both have a red shift in comparison with pure CdTe NCs and the PPV precursor. The red shift of the CdTe NCs is due to the aggregation during the assembly process which is in accordance with the TEM image, while the red shift of the PPV precursor results from the change in chain conformation caused by the electrostatic forces. Interestingly, two peaks appear around $450 \mathrm{~nm}$. It may be that the CdTe NCs reduce the surface charge of the PPV precursor chains, which increases the possibility of the further approach of the chains. Therefore, the PPV precursor has a considerable degree of conformational freedom [36] and the two peaks are assigned to different chain conformations. From Fig. 7(b), the CIE coordinate of the white-light emitting solution is $(0.337,0.332)$, almost the same as the coordinates of balanced white-light emission $(0.333,0.333)$; the insert is the photo of white-light emitting solution under illumination by UV light. We obtain the white-light emission because we solve the two problems often encountered when using NCs to prepare such materials. Firstly, the electrostatic interaction between polymer and CdTe NCs can effectively stabilize the NCs, which avoids the FRET between NCs [45-47]. Secondly, there is no FRET between the polymer and CdTe NCs, which ensures the integration of the properties of CdTe NCs and 
polymer [48, 49]. White-light emission materials prepared using NCs are often unstable. However, our system presents excellent stability due to the introduction of the PVA matrix. We monitored the changes of the PL spectrum of the PPV precursor/CdTe NCs/PVA composite over time. The stability of the pure white-light emitting solution is illustrated in Fig. 8; the chromaticity coordinates remained in the whitelight region over several weeks after it was prepared. Due to the electrostatic interactions, more defects appear on the surface of CdTe NCs, which led to the CdTe NCs being easily photooxidized and a decrease of the PL intensity. However, this decrease is not very marked because of the introduction of the PVA, and both the peak shapes and peak positions in the PL spectra show little change over time, which indicates the excellent stability of the PPV precursor/CdTe NCs/PVA composites.

\section{Conclusion}

We have developed an easy method to prepare materials with emission color tunable from 400 to $800 \mathrm{~nm}$. The introduction of the PVA matrix not only improved the compatibility between the PPV precursor and CdTe NCs and hence enhanced the stability of the system, but also maintained appropriate electrostatic interactions, which prevented the FRET between the PPV precursor and CdTe NCs and realized the integration of their properties. The usage of singlecolored CdTe NCs further avoided the FRET process

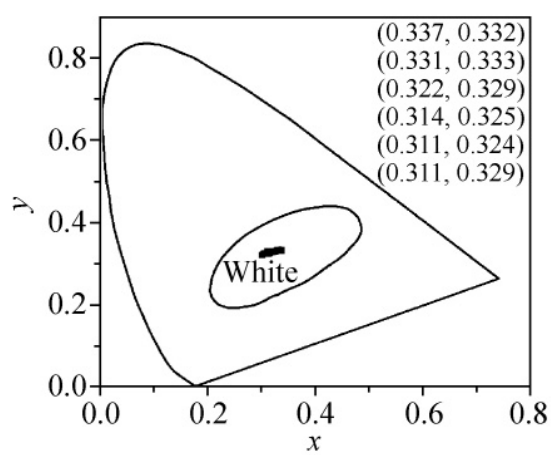

(a)

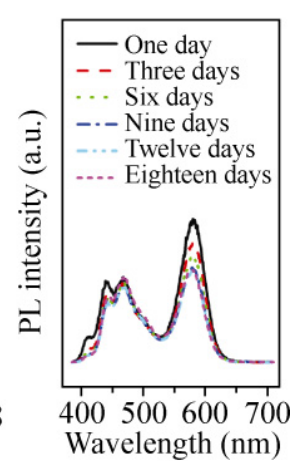

(b)
Figure 8 (a) The change of the chromaticity coordinate graph of the pure white-light emitting solution in the three weeks following its preparation. (b) Variation of the spectra of the pure white-light emitting solution with storage time after preparation which is often present in systems containing mixtures of CdTe NCs emitting different colors. Furthermore, the chromaticity coordinates of the composite solutions can be easily changed by tuning the size and amount of CdTe NCs. Therefore, luminescent materials with almost any chromaticity coordinates in the visible light range can be obtained through our method including pure white-light emitting materials with the CIE coordinates of $(0.337,0.332)$, which could find applications in illumination and background light devices.

\section{Acknowledgements}

This work was supported by National Natural Science Foundation of China (NSFC) (Nos. 20804008, 20974038, 50973039, and 20921003), National Program on Key Basic Research Project (973 Program) (Nos. 2007CB936402 and 2009CB939701), and the China Postdoctoral Science Foundation (No. 20090450139).

Electronic Supplementary Material: The structure of the PPV precursor, and UV-vis absorption and PL spectra of the PPV precursor/PVA mixture are available in the online version of this article at http: //dx.doi.org/ $10.1007 / \mathrm{s} 12274-010-0010-\mathrm{z}$ and is accessible free of charge.

Open Access: This article is distributed under the terms of the Creative Commons Attribution Noncommercial License which permits any noncommercial use, distribution, and reproduction in any medium, provided the original author(s) and source are credited.

\section{References and Notes}

[1] Arakawa, Y.; Sakaki, H. Multidimensional quantum well laser and temperature dependence of its threshold current. Appl. Phys. Lett. 1982, 40, 939-941.

[2] Murray, C. B.; Norris, D. J.; Bawendi, M. G. Synthesis and characterization of nearly monodisperse $\mathrm{CdE}(\mathrm{E}=$ sulfur, selenium, tellurium) semiconductor nanocrystallites. $J$. Am. Chem. Soc. 1993, 115, 8706-8715.

[3] Klimov, V. I.; Mikhailovsky, A. A.; Xu, S.; Malko, A.; Hollingsworth, J. A.; Leatherdale, C. A.; Eisler, H. J.; Bawendi, M. G. Optical gain and stimulated emission in nanocrystal quantum dots. Science 2000, 290, 314-317.

[4] Colvin, V. L.; Schlamp, M. C.; Allvlsatos, A. P. Lightemitting diodes made from cadmium selenide nanocrystals 
and a semiconducting polymer. Nature 1994, 370, 354-357.

[5] Xie, R. G.; Peng, X. G. Synthesis of Cu-doped InP nanocrystals (d-dots) with $\mathrm{ZnSe}$ diffusion barrier as efficient and color-tunable NIR emitters. J. Am. Chem. Soc. 2009, 131, 10645-10651.

[6] Grieve, K.; Mulvaney, P.; Grieser, F. Synthesis and electronic properties of semiconductor nanoparticles/quantum dots. Curr. Opin. Colloid Interface Sci. 2000, 5, 168-172.

[7] Bruchez, M. Jr.; Moronne, M.; Gin, P.; Weiss, S.; Alivisatos, A. P. Semiconductor nanocrystals as fluorescent biological labels. Science 1998, 281, 2013-2016.

[8] Chan, W. C. W.; Nie, S. M. Quantum dot bioconjugates for ultrasensitive nonisotopic detection. Science 1998, 281, 2016-2018.

[9] Åkerman, M. E.; Chan, W. C. W.; Laakkonen, P.; Bhatia, S. N.; Ruoslahti, E. Nanocrystal targeting in vivo. Proc. Natl. Acad. Sci. USA 2002, 99, 12617-12621.

[10] Qian, H. F.; Dong, C. Q.; Peng, J. L.; Qiu, X.; Xu, Y. H.; Ren, J. C. High-quality and water-soluble near-infrared photoluminescent $\mathrm{CdHgTe} / \mathrm{CdS}$ quantum dots prepared by adjusting size and composition. J. Phys. Chem. C 2007, 111, 16852-16857.

[11] Schroedter, A.; Weller, H. Ligand design and bioconjugation of colloidal gold nanoparticles. Angew. Chem. Int. Ed. 2002, 41, 3218-3221.

[12] Russell, J. T.; Lin, Y.; Böker, A.; Su, L.; Carl, P.; Zettl, H.; He, J. B.; Still, K.; Tangirala, R.; Emrick, T.; Littrell, K.; Thiyagarajan, P.; Cookson, D.; Fery, A.; Wang, Q.; Russell, T. P. Self-assembly and cross-linking of bionanoparticles at liquid-liquid interfaces. Angew. Chem. Int. Ed. 2005, 44, 2420-2426.

[13] Tang, Z. Y.; Zhang, Z. L.; Wang, Y. Glotzer, S. C.; Kotov, N. A. Self-assembly of CdTe nanocrystals into free-floating sheets. Science 2006, 314, 274-278.

[14] Zhang, H.; Cui, Z. C.; Wang, Y.; Zhang, K.; Ji, X. L.; Lü, C. L.; Yang, B.; Gao, M. Y. From water-soluble CdTe nanocrystals to fluorescent nanocrystal-polymer transparent composites using polymerizable surfactants. Adv. Mater. 2003, 15, 777-780.

[15] Agrawal, M.; Rubio-Retama, J.; Zafeiropoulos, N. E.; Gaponik, N.; Gupta, S.; Cimrova, V.; Lesnyak, V.; LópezCabarcos, E.; Tzavalas, S.; Rojas-Reyna, R.; Eychmüller, A.; Stamm, M. Switchable photoluminescence of CdTe nanocrystals by temperature-responsive microgels. Langmuir 2008, 24, 9820-9824.

[16] Wang, J. W.; Tanner, P. A. Upconversion for white light generation by a single compound. J. Am. Chem. Soc. 2010, 132, 947-949.

[17] Carlos, L. D.; Sá Ferreira, R. A.; Pereira, R. N.; Assunção,
M.; de ZeaBermudez, V. White-light emission of aminefunctionalized organic/inorganic hybrids: Emitting centers and recombination mechanisms. J. Phys. Chem. B 2004, 108, 14924-14932.

[18] Sorimachi, H.; Ohta, H.; Tanaka, K.; Uchiki, H. Optical properties of Sn-doped and $\mathrm{Sn}$ :Ce-codoped $\mathrm{CaAl}_{2} \mathrm{~S}_{4}$ and application to white light emitting phosphor. Phys. Status Solidi C 2009, 6, 1145-1148.

[19] Liu, J.; Zhou, Q. G.; Cheng, Y. X.; Geng, Y. H.; Wang, L. X.; Ma, D. G.; Jing, X. B.; Wang, F. S. The first single polymer with simultaneous blue, green, and red emission for white electroluminescence. Adv. Mater. 2005, 17, 2974-2978.

[20] Romero-Nieto, C.; Durben, S.; Kormos, I. M.; Baumgartner, T. Simple and efficient generation of white light emission from organophosphorus. building blocks. Adv. Funct. Mater. 2009, 19, 3625-3631.

[21] Wang, R. B.; Peng, J.; Qiu, F.; Yang, Y. L.; Xie, Z. Y. Simultaneous blue, green, and red emission from diblock copolymer micellar films: A new approach to white-light emission. Chem. Commun. 2009, 6723-6725.

[22] Park, S.; Kwon, J. E.; Kim, S. H.; Seo, J.; Chung, K.; Park, S. Y.; Jang, D. J.; Medina, B. M.; Gierschner, J.; Park, S. Y. A white-light-emitting molecule: Frustrated energy transfer between constituent emitting centers J. Am. Chem. Soc. 2009, 131, 14043-14049.

[23] Chen, H. C.; Hung, C. Y.; Wang, K. H.; Chen, H. L.; Fann, W. S.; Chien, F. C.; Chen, P.; Chow, T. J.; Hsu, C. P.; Sun, S. S. White-light emission from an upconverted emission with an organic triplet sensitizer. Chem. Commun. 2009, 4064-4066.

[24] Liu, J.; Zhou, Q. G.; Cheng, Y. X.; Geng, Y. H.; Wang, L. X.; Ma, D. G.; Jing, X. B.; Wang, F. S. White electroluminescence from a single-polymer system with simultaneous two-color emission: Polyfluorene as the blue host and a 2,1,3benzothiadiazole derivative as the orange dopant on the main chain. Adv. Funct. Mater. 2006, 16, 957-965.

[25] Sapra, S.; Mayilo, S.; Klar, T. A.; Rogach, A. L.; Feldmann, J. Bright white-light emission from semiconductor nanocrystals: By chance and by design. Adv. Mater. 2007, 19, 569-572.

[26] Bowers, M. J. II; McBride, J. R.; Garrett, M. D.; Sammons, J. A.; Dukes, A. D. III; Schreuder, M. A.; Watt, T. L.; Lupini, A. R.; Pennycook, S. J.; Rosenthal, S. J. Structure and ultrafast dynamics of white-light-emitting CdSe nanocrystals. J. Am. Chem. Soc. 2009, 131, 5730-5731.

[27] Kar, S.; Biswas, S. White light emission from surface-oxidized manganese-doped $\mathrm{ZnS}$ nanorods. J. Phys. Chem. C 2008, 112, 11144-11149.

[28] Burroughes, J. H.; Bradley, D. D. C.; Brown, A. R.; Marks, R. N.; Mackay, K.; Friend, R. H.; Burns, P. L.; Holmes, A. B. Light-emitting diodes based on conjugated polymers. Nature 
1990, 347, 539-541.

[29] Wienk, M. M.; Kroon, J. M.; Verhees, W. J. H.; Knol, J.; Hummelen, J. C.; van Hal, P. A.; Janssen, R. A. J. Efficient methano[70]fullerene/MDMO-PPV bulk heterojunction photovoltaic cells. Angew. Chem. Int. Ed. 2003, 42, 3371-3375.

[30] Bradley, D. D. C. Precursor-route poly(p-phenylenevinylene): Polymer characterisation and control of electronic properties. J. Phys D: Appl. Phys. 1987, 20, 1389-1410.

[31] Shim, H. S.; Na, S. I.; Nam, S. H.; Ahn, H. J.; Kim, H. J.; Kim, D. Y.; Kim, W. B. Efficient photovoltaic device fashioned of highly aligned multilayers of electrospun $\mathrm{TiO}_{2}$ nanowire array with conjugated polymer. Appl. Phys. Lett. 2008, 92, 183107.

[32] Losurdo, M.; Giangregorio, M. M.; Capezzuto, P.; Cardone, A.; Martinelli, C.; Farinola, G. M.; Babudri, F. F.; Naso, B.; Büchel, M.; Bruno, G. Blue-gap poly(p-phenylene vinylene)s with fluorinated double bonds: Interplay between supramolecular organization and optical properties in thin films. Adv. Mater., 2009, 21, 1115-1120.

[33] Chang, W. P.; Whang, W. T. A study of the relationship between the electroluminescence characteristics and compositions of PPV-PVA-based polymers. Polymer, 1996, 37, 3493-3499.

[34] He, G. F.; Li, Y. F.; Liu, J.; Yang, Y. Enhanced electroluminescence using polystyrene as a matrix. Appl. Phys. Lett., 2002, 80, 4247-4249.

[35] Vallée, R. A. L.; Cotlet, M.; Hofkens, J.; De Schryver, F. C. Spatially heterogeneous dynamics in polymer glasses at room temperature probed by single molecule lifetime fluctuations. Macromolecules 2003, 36, 7752-7758.

[36] Ravi Kishore, V. V. N.; Kokane, S.; Narasimhan, K. L.; Periasamy, N. Time resolved photoluminescence spectra of PPV film: Heterogeneity and excited state relaxation. Chem. Phys. Lett. 2004, 386, 118-122.

[37] Vijila, C.; Westerling, M.; Aarnio, H.; Österbacka, R.; Huang, C.; Chen, Z. K.; Zhang, X. H.; Zhu, F. R.; Jin, C. S. Nature and dynamics of photoexcited states in an electroluminescent poly(phenylene vinylene-co-fluorenylene vinylene)-based $\pi$-conjugated polymer. J. Photochem. Photobiol. A: Chem. 2008, 199, 358-362.

[38] Colaneri, N. F.; Bradley, D. D. C.; Friend, R. H.; Burn, P. L.; Holmes, A. B.; Spangler, C. W. Photoexcited states in poly( $p$-phenylene vinylene): Comparison with trans, transdistyrylbenzene, a model oligomer. Phys. Rev. B 1990, 42, 11670-11681.
[39] Bjorklund, T. G.; Lim, S. H.; Bardeen, C. J. The optical spectroscopy of poly( $p$-phenylene vinylene)/polyvinyl alcohol blends: From aggregates to isolated chromophores. Synth. Met. 2004, 142, 195-200.

[40] Jarzab, D.; Cordella, F.; Lenes, M.; Kooistra, F. B.; Blom, P. W. M.; Hummelen, J. C.; Loi, M. A. Charge transfer dynamics in polymer-fullerene blends for efficient solar cells. J. Phys. Chem. B 2009, 113, 16513-16517.

[41] Edman, L.; Mets, Ü.; Rigler, R. Conformational transitions monitored for single molecules in solution. Proc. Natl. Acad. Sci. USA 1996, 93, 6710-6715.

[42] Al Attar, H. A.; Monkman, A. P. Effect of surfactant on water-soluble conjugated polymer used in biosensor. J. Phys. Chem. B 2007, 111, 12418-12426.

[43] Sun, H. Z.; Zhang, H.; Zhang, J. H.; Ning, Y.; Yao, T. J.; Bao, X.; Wang, C. L.; Li, M. J.; Yang, B. Effect of electrostatic interactions on the photophysical properties of the composites of CdTe nanocrystals and carbazole-containing polymers. J. Phys. Chem. C 2008, 112, 2317-2324.

[44] Sun, H. Z.; Zhang, H.; Zhang, J. H.; Wei, H. T.; Ju, J.; Li, M. J.; Yang, B. White-light emission nanofibers obtained from assembling aqueous single-colored CdTe NCs into a PPV precursor and PVA matrix. J. Mater. Chem. 2009, 19, 6740-6744.

[45] Müller, F.; Götzinger, S.; Gaponik, N.; Weller, H.; Mlynek, $\mathrm{J}$; Benson, O. Investigation of energy transfer between CdTe nanocrystals on polystyrene beads and dye molecules for FRET-SNOM applications. J. Phys. Chem. B 2004, 108, 14527-14534.

[46] Willard, D. M.; Carillo, L. L.; Jung, J.; Orden, A. V. $\mathrm{CdSe}-\mathrm{ZnS}$ quantum dots as resonance energy transfer donors in a model protein-protein binding assay. Nano Lett. 2001, 1, 469-474.

[47] Sun, H. Z.; Zhang, J. H.; Zhang, H.; Xuan, Y.; Wang, C. L.; Li, M. J.; Tian, Y.; Ning, Y.; Ma, D. G.; Yang, B.; Wang, Z. Y. Pure white-light emission of nanocrystal-polymer composites. ChemPhysChem 2006, 7, 2492-2496.

[48] Jiang, G. X.; Susha, A. S.; Lutich, A. A.; Stefani, F. D.; Feldmann, J.; Rogach, A. L. Cascaded FRET in conjugated polymer/quantum dot/dye-labeled DNA complexes for DNA hybridization detection. ACS Nano 2009, 3, 4127-4131.

[49] Lee, J.; Kim, H. J.; Chen, T.; Lee, K.; Kim, K. S.; Glotzer, S. C.; Kim, J.; Kotov, N. A. Control of energy transfer to CdTe nanowires via conjugated polymer orientation. J. Phys. Chem. C 2009, 113, 109-116. 